\title{
Robust Direction of Arrival Estimation using Subspace Methods
}

\author{
Ch. Kusuma Kumari, PhD \\ GVP College of \\ Engineering (A), \\ Visakhapatnam
}

\author{
G. Radha Kumari \\ GVP College of \\ Engineering $(A)$ \\ Visakhapatnam.
}

\begin{abstract}
Direction of arrival (DOA) estimation is one of the focal problems in the fields of Wireless communications, Radar, Sonar, Radio Astronomy and Seismology. The main objective of the DOA estimation is to obtain the desired signals direction as well as the interference signals direction based on the data received from the array sensor at the base station. In the literature various techniques are available among which the two high resolution algorithms namely MUSIC (MUltiple SIgnal Classification) and ESPRIT (Estimation of Signal Parameters via Rotational Invariance Technique) are used for direction of arrival estimation in this paper. The work presented in this paper deals with EMD application to the DOA estimation as a preprocessing technique. The key feature of EMD is to decompose a signal into sum of intrinsic mode functions (IMF's) with a final residue. This technique separately de-noises the rows of the array data matrix where each row corresponds to the output of a particular array sensor. Especially in low-SNR conditions, the estimation performance of MUSIC and ESPRIT algorithms is enhanced significantly when de-noising is given to array data matrix prior to DOA estimation stage.
\end{abstract}

\section{Keywords}

Direction of arrival (DOA), Multiple Signal Classification (MUSIC), Estimation of Signal Parameters via Rotational Invariance Technique (ESPRIT), Empirical Mode Decomposition (EMD), Intrinsic Mode Function (IMF).

\section{INTRODUCTION}

Antenna array design has received much attention in the last few decades. It is one of the areas of research in the wideranging field of signal processing, and focuses on the problem of estimating signal parameters from the data received at the antenna array. In general, the parameters are the signal contents, the angles of arrival and the power. Spectral estimation techniques have the capability to select desired frequency components out of a composite signal in the presence of noise and interference [1]. DOA estimation plays an important role in array signal processing, because both the base station and mobile station can use manifold antenna elements enhancing the capacity and throughput of the system significantly.

In recent years, the detection of a signal from a particular direction has received noteworthy significance in the applications like defence, wireless mobile communications, radar, sonar and tracking targets [2]. In commercial applications, the significance of DOA estimation is essential to spot the direction of an emergency telephone call in order to dispatch a rescue squad to the right location. In mobile communications, the major intent of DOA estimation is to estimate the direction of the desired user's signals and also the directions of interferer's signals based on the received data at the sensor array base-station. In defence applications, the role of DOA estimation is to recognize the direction of possible threats. Different DOA estimation algorithms available in the literature are Bartlett, Capon, Min-norm, MUSIC, and ESPRIT. However MUSIC and ESPRIT algorithms can be considered to be the high resolution and exact methods that are commonly used in the smart antenna design [3] and are used in this paper.

It is well-known that in real scenario of data analysis the signals do not exist without noise, considered negligible for high SNR. Yet, there are conditions in which the noise significantly corrupts the signal, and it must be removed in order to proceed with further data analysis. The procedure for noise removal is referred to as signal de-noising or simply denoising. There is a large range of applications in which denoising is important. Some of them are medical signal analysis, data mining, and radio astronomy. The motivation to this paper is to achieve robust DOA estimation by reducing the effect of noise. Hence EMD is treated as a de-noising technique which is capable of decomposing a signal into sum of intrinsic mode functions (IMF) [4].

This paper is structured as follows. Section 2 describes the receiving signal model. Section 3 presents DOA estimation using MUSIC and ESPRIT algorithms. Empirical Mode Decomposition (EMD) as a de-noising technique is explained in section 4. The proposed method is explained in section 5. Simulation results and discussions are given in section 6 . Finally the conclusion is given in section7.

\section{SIGNAL MODEL}

The signal model shown in Fig.1 describes $\boldsymbol{D}$ signals that are arriving from $D$ different directions and they are received by $N$ array elements. Each received signal $\mathrm{X}_{\mathrm{N}}(\mathrm{K})$ includes zero mean white Gaussian noise. Time is represented by the $k^{\text {th }}$ sample, thus the array output $\mathrm{X}(\mathrm{K})$ is given by

$$
\begin{aligned}
& X(k)=\left[a\left(\theta_{1}\right), a\left(\theta_{2}\right) \ldots a\left(\theta_{D}\right)\right]\left[\begin{array}{l}
S_{1}(k) \\
S_{2}(k) \\
\vdots \\
S_{D}(k)
\end{array}\right]+n(k) \\
& X(k)=A * S(k)+n(k) \ldots \ldots \ldots \ldots \ldots \ldots \ldots(1) \\
& S(k)=\text { incident complex signals vector at time } k \\
& n(k)=\text { vector of at each array element } \mathrm{N}
\end{aligned}
$$




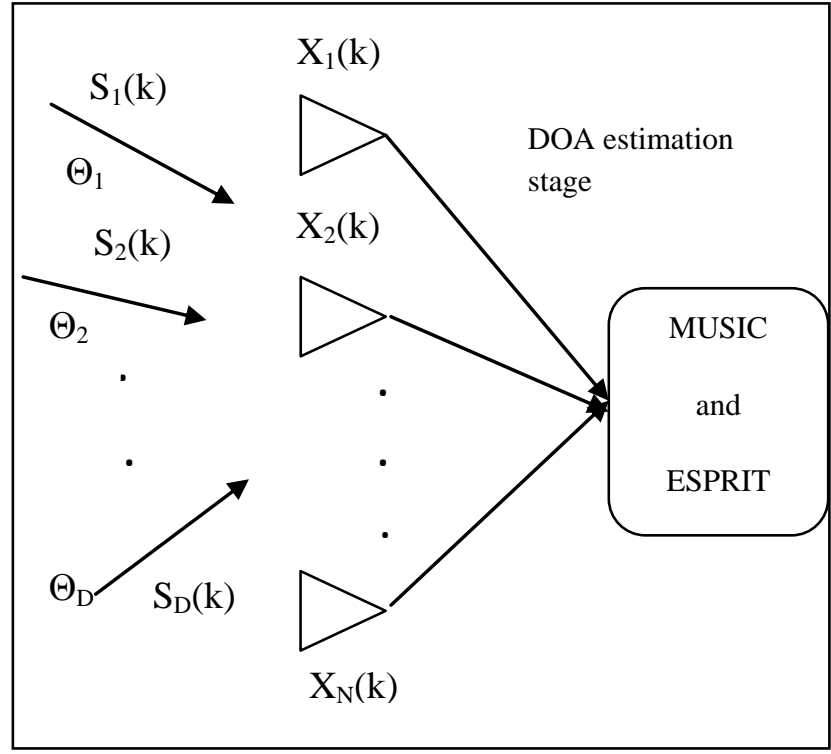

Fig 1: Receive array with incident plane waves from various directions

$A=\left[a\left(\theta_{1}\right), a\left(\theta_{2}\right), \ldots a\left(\theta_{N}\right)\right]$, an $(N \mathrm{X} D)$ matrix of steering vectors.

$a\left(\theta_{\mathrm{i}}\right)=\mathrm{N}$-element array steering vector for the $\theta_{i}$ direction of arrival

$a\left(\theta_{i}\right)=\left[1-e^{\frac{j 2 \Pi d \sin \theta i}{\lambda}} \ldots . . . e^{\frac{j 2 \Pi d(N-1) \sin \theta i}{\lambda}}.\right]$ where $, i=1,2,3, \ldots . D$

The calculations are based on instances of the incoming signal because of the time varying nature of arriving signals. Since the matrix of steering vectors is changing with time, the corresponding arrival angles will also change. The correlation matrix of the array and the source correlation matrix are found by the expected value of the respective absolute values squared. i.e.

The noise covariance matrix is $R_{n n}=E\left[n(t) n^{H}(t)\right]$

The source covariance matrix is

$$
R_{s s}=E\left[s(t) s^{H}(t)\right]
$$

The array output covariance matrix is

$R_{x x}=E\left[X(t) X^{H}(t)\right]$

Thus, $R_{x x}=A R_{s s} A^{H}+R_{n n}$

Correlation matrix is often referred to as the covariance matrix if the mean values of the signals and noise are zero. Given -array elements with $D$ narrowband signal sources with uncorrelated noise. In [5] the following assumptions on the correlation matrix are considered and the same are also considered in this paper. First assumption is that, $\mathrm{R}_{\mathrm{xx}}$ is an $N \mathrm{X} N$ Hermitian matrix. A Hermitian matrix is equal to its complex conjugate transpose i.e., $R_{X X}=R_{X X}^{H}$. The correlation matrix of the array has $N$ Eigen values $\lambda_{1}, \lambda_{2}, \ldots \ldots . . \lambda_{N}$ along with $\mathrm{N}$ associated Eigen vectors $E=\left[e_{1} e_{2} e_{3} \ldots \ldots . e_{N}\right]$. Second assumption is, if the Eigen values are sorted from smallest to largest and the matrix
E is divided into two subspaces such that $E=E_{N} E_{S}$. The subspace ENis called the noise subspace and is constructed by the $(N-D)$ Eigen vectors related with the noise. For uncorrelated noise, the Eigen values are given as $\lambda_{1}=\lambda_{2}=\ldots \lambda_{N-D}=\sigma_{n}^{2}$.The subspaceES is called the signal subspace and is constructed by the $D$ Eigen vectors related with the arriving signals. The noise subspace $\mathrm{E}_{\mathrm{N}}$ is an $N \mathrm{X}(N-D)$ matrix. The signal subspace ES is a $N \mathrm{X} D$ matrix.

The objective of DOA estimation methods is to describe a function which gives an indication of the angles of the incoming signal based upon maxima vs. angle. This function is conventionally called the pseudo spectrum. The unit of the pseudo spectrum is in energy or in watts.

\section{DOA ESTIMATION ALGORITHMS}

The DOA estimation algorithms are categorized as conventional and subspace algorithms. The Bartlett and Capon (also called as Minimum Variance Distortionless Response) are conventional type algorithms which are highly reliant on the physical size of the array aperture which results in deprived resolution and exactness. MUSIC and ESPRIT methods are categorized under subspace based methods based on the Eigen decomposition [6] which are more precise and gives high resolution and not limited to physical size of the array aperture [7].

\subsection{MUSIC Algorithm}

MUltiple SIgnal Classification (MUSIC) is one of the popular high resolution techniques that give estimation of the DOA which was given by Statesmen [8]. This algorithm is based on exploiting the Eigen structure of input covariance matrix. The incident complex signals are somewhat correlated creating non diagonal signal correlation matrix. The algorithm is used to describe the techniques involved in obtaining the parameters of various wave fronts arriving at the antenna array based on the calculations made on the received signal at the sensor array. MUSIC algorithm divides the decomposition of the input covariance matrix into two orthogonal matrices, i.e., signal-subspace and noise-subspace in which the noise subspace is used for finding the Direction of arrival. The algorithm is summarized as following:

1. The array correlation matrix is found by

$$
R_{x x}=A R_{s s} A^{H}+\sigma_{n}^{2}
$$

2. The Eigen values and Eigen vectors for $R_{x x}$ are calculated and sorted them in ascending order.

3. Last ' $D$ ' Eigen vectors are corresponding to the signals and First ' $N-D$ ' Eigen vectors are corresponding to the noise.

4. The $N \mathrm{X}(N-D)$ dimensional subspace spanned by the Eigen vectors corresponding to the noise is formed such that $E_{N}=\left[e_{1} e_{2} \ldots . . e_{N-D}\right]$

5. The MUSIC pseudo spectrum is obtained by

$$
P_{\text {MUSIC }}(\theta)=\frac{1}{\left|a(\theta)^{H} E_{N} E_{N}^{H} a(\theta)\right|}
$$


The DOA angle is estimated by the peaks in the obtained pseudo spectrum.

\subsection{ESPRIT Algorithm}

Although MUSIC was the first of the high-resolution algorithm to correctly exploit the underlying data model of narrow band signals in additive noise, the algorithm has several limitations including the fact that complete knowledge of the array manifold is required and the search over parameter space is computationally very expensive [8].

ESPRIT is similar to MUSIC in that it correctly exploits the underlying data model while manifesting significant advantages over MUSIC. ESPRIT was first proposed by ROY and KAILATH [9] .The goal of the ESPRIT technique is to exploit the rotational invariance in the signal subspace which is created by two arrays with translational invariance structure as shown in Fig 2 ESPRIT assumes multiple identical arrays called doublets. These can be separate arrays or composed of sub arrays of one larger array. It is important that these arrays are displaced translationally but not rotationally. Useful summaries of this technique are given by both Godara [10] and Liberti and Rappaport [11].

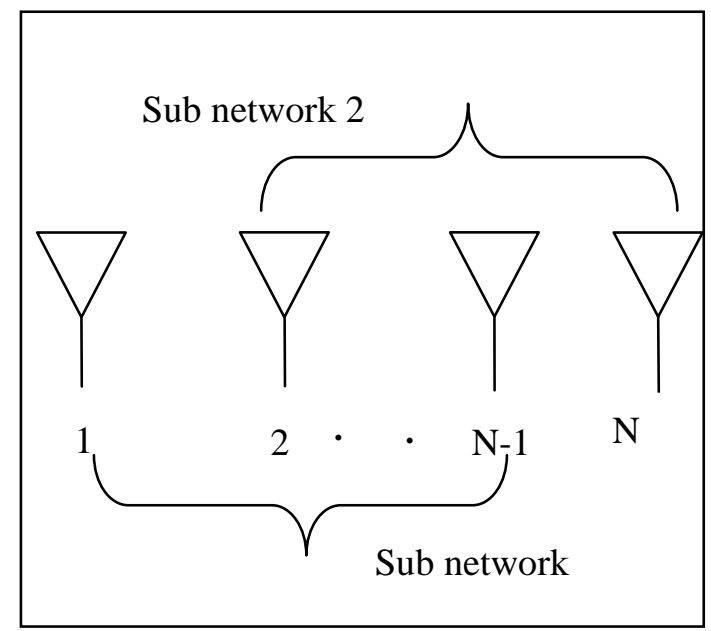

Fig 2: Doublet composed of identical displaced arrays

The steps of the algorithm are summarized as follows:

1. Perform Eigen decomposition on Rxx.

2. Estimate the correlation matrix Rxx.

3. Obtain the signal subspace estimate $\mathrm{Ex}$ and decompose it into sub-array matrices $\mathrm{E}_{1}$ and $\mathrm{E}_{2}$.

4. Compute the Eigen decomposition of the signal subspace and partition it into sub-matrices.

$$
\mathrm{C}=\left[\begin{array}{c}
E_{1}^{H} \\
E_{2}^{H}
\end{array}\right]\left[\begin{array}{ll}
\mathrm{E}_{1} & \mathrm{E}_{2}
\end{array}\right]=\mathrm{E}_{\mathrm{C}} \wedge \mathrm{E}_{\mathrm{C}}^{\mathrm{H}}
$$

5. Partition Ec into four $\mathrm{D} \times \mathrm{D}$ sub matrices such that

$$
\mathrm{E}_{\mathrm{C}}=\left[\begin{array}{ll}
\mathrm{E}_{11} & \mathrm{E}_{12} \\
\mathrm{E}_{21} & \mathrm{E}_{22}
\end{array}\right]
$$

6. Estimate the rotational operator $\psi$ by

$$
\Psi=\mathrm{E}_{12} \mathrm{E}_{22}^{-1}
$$

7. Calculate the Eigen values of $\psi$

8. Estimate the angle of arrival using

$$
\theta_{\mathrm{i}}=\sin ^{-1}\left(\frac{\arg \left(\lambda_{i}\right)}{\mathrm{kd}}\right), \quad \mathrm{i}=1,2, \ldots \ldots \ldots \ldots, \mathrm{D}
$$

\section{EMPIRICAL MODE DECOMPOSITION}

EMD is relatively unconventional method in Signal Processing proposed by Huang et al. [4]. It is an adaptive signal analysis method applicable to time-frequency analysis of nonlinear and non stationary signals. EMD decomposes the given signal into a finite number of zero-mean functions, called Intrinsic Mode Functions (IMFs). These IMF's are obtained as the combination of amplitude and frequency modulated sinusoids.

An IMF is defined as a function that satisfies the following requirements:

1. In the entire data set, the number of extrema and the number of zero-crossings must be equal or differ at most by 1 .

2. The mean value of the envelope defined by the local maxima and the local minima at any point is zero.

Instead of constant amplitude and frequency in a simple harmonic component, an IMF can have variableamplitude and frequency along the time axis. The procedure of extracting an IMF is called "sifting". IMF generation process for a given signal is shown in Fig 3. 


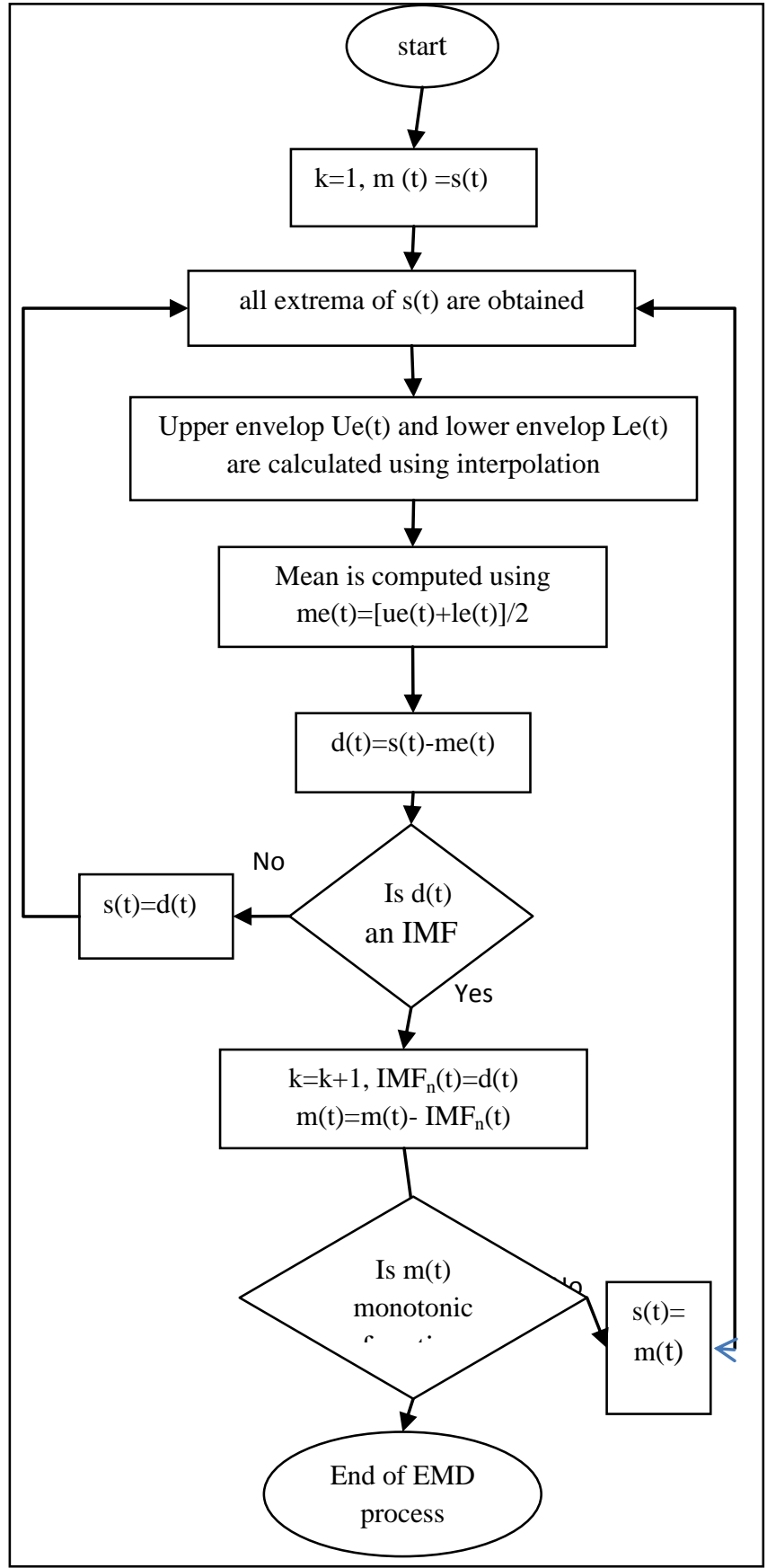

Fig 3: Flow diagram of EMD process

\section{EMD BASED DOA ESTIMATION}

Robust scheme for DOA estimation using Empirical Mode Decomposition (EMD) algorithm is shown in Fig 4. EMD is given for pre-processing prior to the DOA estimation stage. The algorithm separately de-noises the rows of the array data matrix where each row corresponds to the output of a particular array sensor, i.e. the signals $X_{1}(K), X_{2}(K) \ldots X_{N}(K)$ are given to EMD de-noising.

$$
X i(K)=A . S(K)+n(K), \text { for } \mathrm{i}=1,2, . . \mathrm{N} .
$$

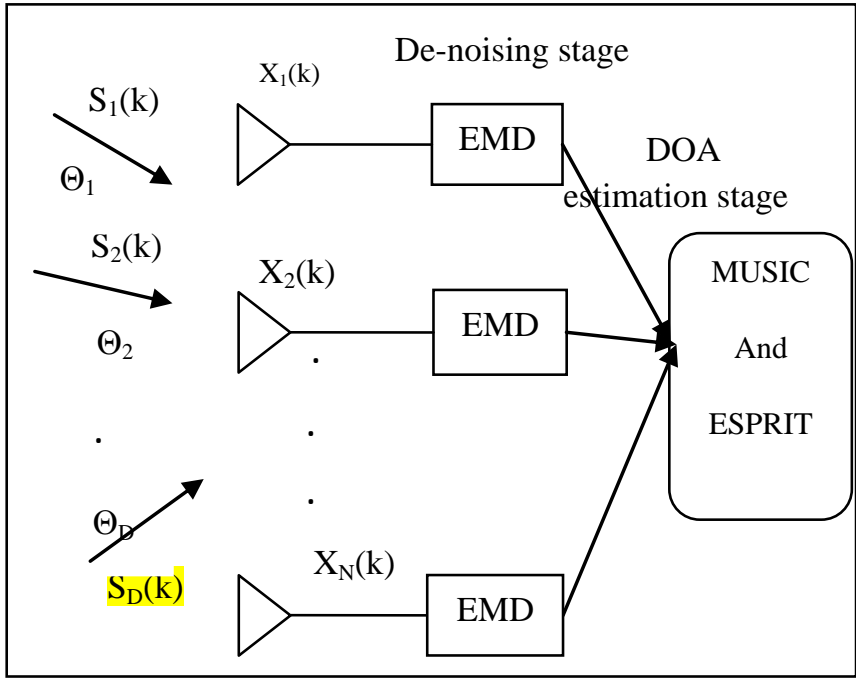

Fig.4: Receive array prior to de-noising stage

The noise corrupted signals $X i(K)$ (for $\mathrm{i}=1,2, . . \mathrm{N}$ ) are given to EMD and the respective IMFs are generated by decomposition procedure. Generally a noise signal is characterized by high frequency components [12]. Thus, initially generated IMFs are treated as noise IMFs and are discarded and the remaining IMF's are summed along with the residue. The de-noised signal is then applied to the MUSIC and ESPRIT algorithms for estimating the direction of arrival.

\section{SIMULATION RESULTS AND DISCUSSIONS}

The MUSIC and ESPRIT techniques for DOA estimations are simulated using MATLAB tool. The performance of these algorithms has been analyzed at various signal to noise ratio conditions. The numerical example considered in this paper is with $\mathrm{N}=6$ array elements separated by a distance of $\lambda / 2$. Additive white Gaussian noise is considered with 0.1 variance. The simulation has been run for two incoming signals from two different angles $\theta 1=-250$ and $\theta 2=350$. These two signals are considered to have equal amplitudes. The effect of changing the SNR with four different values $(10,20$,10,-20) dB is shown in Fig.5 and Fig. 6.

The pseudo spectrum of MUSIC algorithm is shown in Fig 6 . The peaks are obtained from the equation from MUSIC algorithm. The red colour plot represents the peaks obtained for $\mathrm{SNR}=-20 \mathrm{~dB}$ and the blue colour plot represents the peaks obtained for $S N R=20 \mathrm{~dB}$. For high values of $S N R$, the algorithm exhibits good performance whereas for very low SNR value $(-20 \mathrm{~dB})$ MUSIC algorithm is not able to detect the peaks or the true angles. 


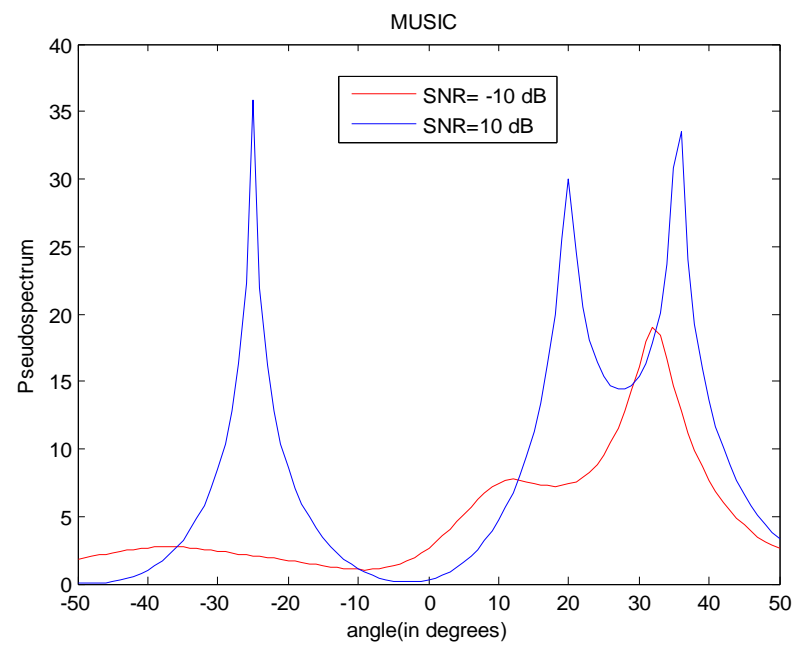

Fig 5: The effect of variation in SNR $(10,-10)$ on MUSIC algorithm

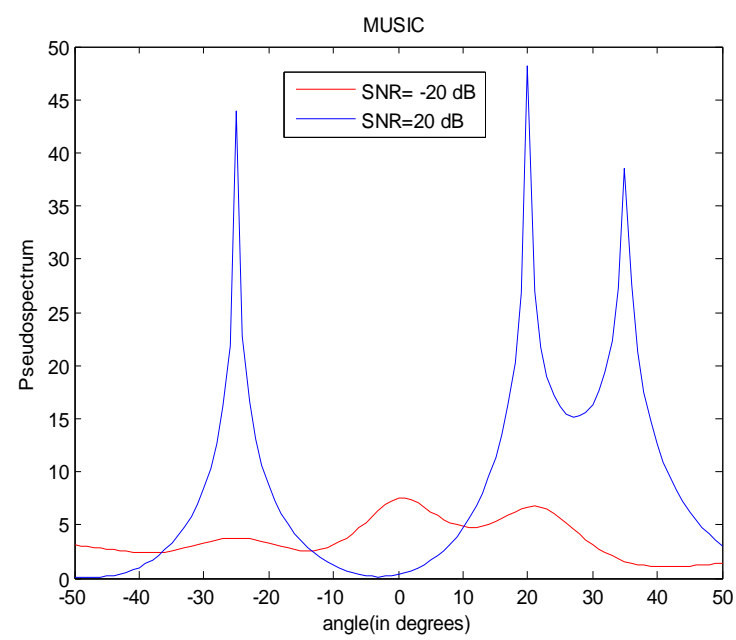

Fig 6: The effect of variation in SNR $(20,-20)$ on MUSIC algorithm

The MUSIC and EMD based MUSIC algorithms are compared and shown in Fig.7 and Fig 8. The red colour plot indicates the EMD based MUSIC algorithm and the blue colour plot indicates the MUSIC algorithm. For SNR = $10 \mathrm{~dB},-20 \mathrm{~dB}$ MUSIC algorithm gives the approximated values whereas EMD-MUSIC is able to give the values same as the true angles.

Table 1 gives the comparison of the angles of arrival between MUSIC algorithm and EMD-based MUSIC algorithm with respect to various SNR values. It is evident that for the high values of SNR (say $10 \mathrm{~dB}, 20 \mathrm{~dB}$ ) MUSIC algorithm exhibits good performance. But the performance of the MUSIC algorithm is degraded with the increase of noise. Using EMD as a denoising stage prior to the DOA stage, the performance of DOA estimation algorithms is improved. From the numerical example it can be observed that even at low SNR condition i.e., at $-20 \mathrm{~dB}$ the signal is estimated. So EMD based denoising enhances the resolution in DOA estimation [13].

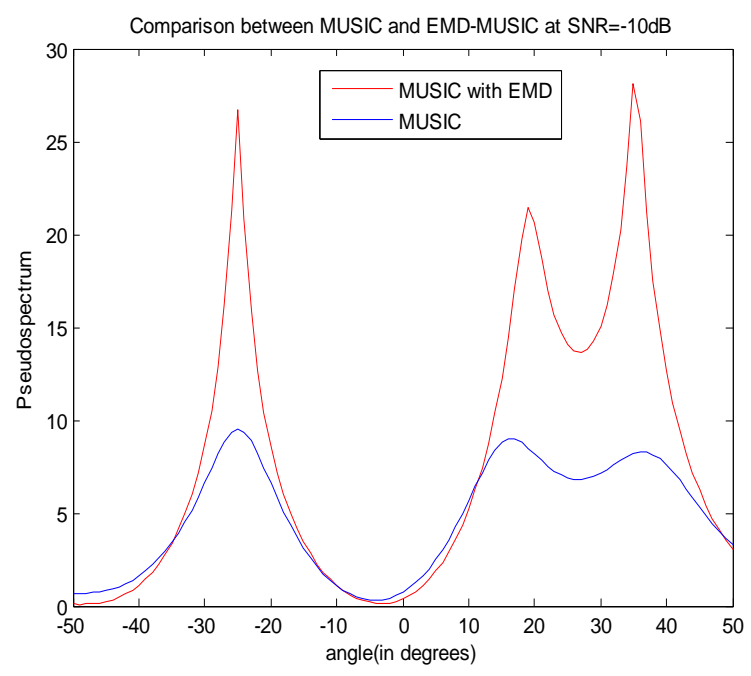

Fig 7: Comparison between MUSIC and EMD-MUSIC spectrum for $\mathrm{SNR}=-\mathbf{1 0 d B}$

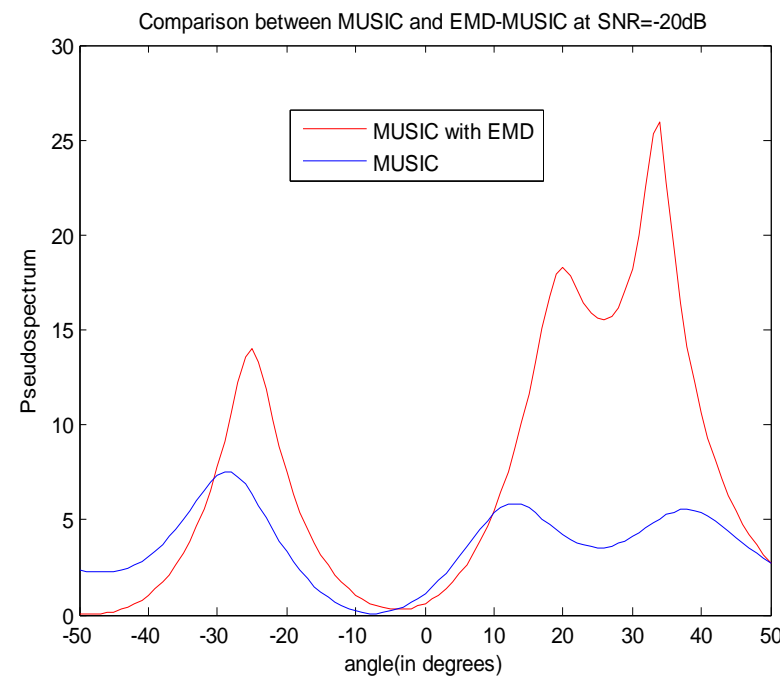

Fig 8: Comparison between MUSIC and EMD-MUSIC spectrum for $\mathrm{SNR}=-\mathbf{2 0 d B}$

Table 2 gives the comparison of the angles of arrival between ESPRIT algorithm and EMD-based ESPRIT algorithm with respect to various SNR values. It is evident that for the high values of SNR (say $10 \mathrm{~dB}, 20 \mathrm{~dB}$ ) ESPRIT algorithm exhibits good performance. But the performance of the ESPRIT algorithm is degraded with the increase of noise. Using EMD as a denoising stage prior to the DOA estimation stage the performance of DOA estimation algorithms is improved. From the numerical example it can be observed that even at low SNR condition i.e., at $-20 \mathrm{~dB}$ the signal is estimated. So EMD based denoising enhances the resolution in DOA estimation. The results show that with use of EMD technique as a pre- processing technique, the estimation performance of the MUSIC and ESPRIT algorithms has been improved. The proposed method for DOA estimation is able to estimate the incoming angles accurately at very low SNR conditions considered in the numerical example. 
Table 1:Angle estimation of MUSIC at various SNR's

\begin{tabular}{|c|c|c|c|}
\hline $\begin{array}{c}\text { SNR } \\
\text { (in dB) }\end{array}$ & $\begin{array}{c}\text { TRUE } \\
\text { ANGLE } \\
\text { (in degrees) }\end{array}$ & $\begin{array}{c}\text { MUSIC } \\
\text { Estimated } \\
\text { ANGLE } \\
\text { (in } \\
\text { degre } \\
\text { es) }\end{array}$ & $\begin{array}{c}\text { with EMD } \\
\text { MUSIC } \\
\text { Estimated } \\
\text { ANGLE } \\
\text { (in } \\
\text { degre } \\
\text { es) }\end{array}$ \\
\hline \multirow{3}{*}{20} & -25 & -25 & -25 \\
\hline & 20 & 20 & 20 \\
\hline & 35 & 35 & 35 \\
\hline \multirow{3}{*}{10} & -25 & -25 & -25 \\
\hline & 20 & 20 & 20 \\
\hline & 35 & 35 & 35 \\
\hline \multirow{3}{*}{-10} & -25 & -25 & -25 \\
\hline & 20 & 16 & 19 \\
\hline & 35 & 36 & 35 \\
\hline \multirow{3}{*}{-20} & -25 & -29 & -25 \\
\hline & 20 & 13 & 20 \\
\hline & 35 & 38 & 34 \\
\hline
\end{tabular}

Table 2: Angle estimation of ESPRIT at various SNR's

\begin{tabular}{|c|c|c|c|}
\hline $\begin{array}{c}\text { SNR } \\
\text { (in dB) }\end{array}$ & $\begin{array}{c}\text { TRUE } \\
\text { ANGL } \\
\mathbf{E} \\
\text { (in degrees) }\end{array}$ & $\begin{array}{c}\text { ESPRIT } \\
\text { Estimat } \\
\text { ed } \\
\text { ANGLE } \\
\text { (in degrees) }\end{array}$ & $\begin{array}{c}\text { with EMD } \\
\text { ESPRI } \\
\mathbf{T} \\
\text { Estimat } \\
\text { ed } \\
\text { ANGL } \\
\mathbf{E} \\
\text { (in degrees) }\end{array}$ \\
\hline \multirow{3}{*}{20} & -25 & -25 & -25 \\
\hline & 20 & 20 & 20 \\
\hline & 35 & 35 & 35 \\
\hline \multirow{3}{*}{10} & -25 & -25 & -25 \\
\hline & 20 & 20 & 20 \\
\hline & 35 & 35 & 35 \\
\hline \multirow{3}{*}{-10} & -25 & -25 & -25 \\
\hline & 20 & 19 & 20 \\
\hline & 35 & 38 & 35 \\
\hline \multirow{3}{*}{-20} & -25 & -28 & -25 \\
\hline & 20 & 23 & 20 \\
\hline & 35 & 43 & 35 \\
\hline
\end{tabular}

\section{CONCLUSIONS AND FUTURE SCOPE}

This paper presents results of Direction of Arrival estimation using MUSIC and ESPRIT algorithms. The ability to resolve incoming signals accurately and efficiently has been performed based on the effect of signal to noise ratio. To increase the estimation performance, EMD based de-noising scheme is considered. Empirical Mode Decomposition (EMD) is a robust de-noising method for non-linear and nonstationary signals. The results show that the estimation performance of MUSIC and ESPRIT algorithms has been increased significantly when EMD based de-noising is applied prior to DOA estimation stage for the low SNR conditions (for $-10 \mathrm{~dB}$ and $-20 \mathrm{~dB}$ ).

The output obtained at DOA estimation stage in this method is used for adaptive beam forming in the design of smart antenna systems is the future scope of this paper. Also,by using threshold angles with better filtering techniques high resolution of DOA can be achieved. In Electronic Counter Measures (ECM) applications an accurate estimation of direction is needed. By using improved EMD technique it can be obtained.

\section{REFERENCES}

[1]. Moustafa M. Abdalla, Mostafa B. Abuitbel, Mohamed A. Hassan, 2013." Performance evaluation of Direction Of Arrival estimation using music and esprit algorithms for mobile communication systems"IEEE.

[2]. Frank B. Gross,2005. Smart Antennas for Wireless Communications

[3]. Mustafa Abdalla, Khaled A. Madi, and Rajab Farhat, 2012. "Sensitivity Analysis for Direction Of Arrival Estimation Using Capon and Music Algorithms in Mobile Radio Environment", World Academy of Science, Engineering and Technology72, 1756-1761.

[4]. N.E.Huang, Z. Shen, S. R. Long et al.,1998.“The empirical mode decomposition and the Hubert spectrum for nonlinear and non-stationary time series analysis," Proceedings of the RoyalSociety A, 454, 903-995.

[5]. Dr. Md. Rafiqul Islam, Ibrahim A. H. Adam.2009. "Performance Study of Direction of Arrival (DOA) Estimation Algorithms for Linear Array Antenna",IEEE, 268-271.

[6]. Andy VESA, Arpad IOZSA,2010. "Direction - of Arrival Estimation for Uniform Sensor Arrays",IEEE,10, 249-252.

[7]. K.Al-Midfa, "Investigation of Direction-of-Arrival Algorithms".2003.Ph.D. Thesis, University of Bristol, UK,

[8]. Schmidt. R1986. "Multiple Emitter Location and Signal Parameter Estimation," IEEE Transactions on Antenna. Propagation, 2, 276-280.

[9]. Roy, R., and T. Kailath, 1989."ESPRIT-Estimation of Signal Parameters Via Rotational Invariance Techniques," IEEE Transactions,37, 7:984-995.

[10].Godara.L,1997. "Application of Antenna Arrays to Mobile Communications, Part II: Beam-Forming and Direction-of-Arrival Considerations," Proceedings of the IEEE, 85, 8:1195-1245.

[11].Liberti, J., and T. Rappaport, 1999.Smart Antennas forWireless Communications, Prentice Hall, New York.

[12].W.-B. Jin and Y. Jun, 2007."Velocity determination of single sound source based on Doppler Effect," IEEE, 956-961.

[13] Raja Sekhar Yeduri, G.Radha kumari, Dr.Ch.Kusma Kumari,2014. "Robust direction of arrival estimation using multiple signal analysis",International Journal of Computer Applications (0975 - 8887),101,11:17-21. 